\title{
Interlinking motifs and entropy landscapes of statistically interacting particles
}

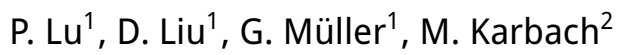 \\ ${ }^{1}$ Department of Physics, University of Rhode Island, Kingston RI 02881, USA \\ 2 Fachbereich Physik, Bergische Universität Wuppertal, 42097 Wuppertal, Germany
}

Received November 28, 2011

The $s=1 / 2$ Ising chain with uniform nearest-neighbor and next-nearest-neighbor coupling is used to construct a system of floating particles characterized by motifs of up to six consecutive local spins. The spin couplings cause the assembly of particles which, in turn, remain free of interaction energies even at high density. All microstates are configurations of particles from one of three different sets, excited from pseudo-vacua associated with ground states of periodicities one, two, and four. The motifs of particles and elements of pseudo-vacuum interlink in two shared site variables. The statistical interaction between particles is encoded in a generalized Pauli principle, describing how the placement of one particle modifies the options for placing further particles. In the statistical mechanical analysis arbitrary energies can be assigned to all particle species. The entropy is a function of the particle populations. The statistical interaction specifications are transparently built into that expression. The energies and structures of the particles alone govern the ordering at low temperature. Under special circumstances the particles can be replaced by more fundamental particles with shorter motifs that interlink in only one shared site variable. Structures emerge from interactions on two levels: particles with shapes from coupled spins and long-range ordering tendencies from statistically interacting particles with shapes.

Key words: Pauli principle, particles with shapes, fractional statistics, Ising model, solitons

PACS: $05.50 .+q, 75.10 .-b$

\section{Introduction}

Condensed matter is an aggregate of interacting particles. The interactions operate in hierarchies regarding strength and range. Atomic nuclei are composed of strongly coupled protons and neutrons. Electrons are bound to atomic nuclei by electromagnetic interactions of widely varying strength. Inner electrons are tightly bound to the nucleus and form ion cores. Some outer electrons may be traded or shared between neighboring ion cores in ionic or valence bonds, respectively. In metallic bonds some outer electrons are mobilized.

Sorting out the diverse, complex, and interrelated phenomena is challenging. The common strategy of many approaches is to transform specific aspects of the strongly interacting ion cores and electrons into more weakly interacting collective modes. The latter share many attributes with fundamental particles including energy-momentum relations, spin, and exclusion statistics. They scatter off each other elastically or inelastically, form bound states, or decay into other modes. The goal of transforming the strongly coupled constituent particles of condensed matter into collective modes that behave like free particles is elusive except under idealized circumstances related to dynamic or kinematic restrictions.

Harmonic lattice vibrations have linear equations of motion. The collective modes exist in superpositions of infinite lifetimes without scattering. Phonons have bosonic statistics. Linear combinations of atomic orbitals produce fermionic counterparts: band electrons [1]. In a model that constrains the kinematics of collective modes to one dimension and limits their dynamics to elastic two-body scattering, the momenta and energies are conserved individually. The collective modes are particles with exclusion statistics determined by the (factorizing) $S$-matrix, analyzed via Bethe ansatz [2-5]. Lattice degrees 
of freedom that are coupled by commuting operators are static. They can be assembled into particles that are free and floating. These particles have definite energies independent of the neighborhood. Their mutual exclusion statistics are exotic [6-8].

This project imposes the kinematic constraint of one dimension and the dynamic constraint of commuting operators. It aims at shedding light on the assembly of structures from interactions and on the emergence of order from these structures within that limited realm. We expect that the results will open doors for situations where the kinematic or dynamic constraints are relaxed. The Ising chain for commuting spin operators $S_{l}^{z}= \pm \frac{1}{2}$ with nearest-neighbor (nn) coupling $J$, next-nearest-neighbor (nnn) coupling $L$, magnetic field $h$, and periodic boundary conditions [9],

$$
\mathscr{H}=\sum_{l=1}^{N}\left[J S_{l}^{z} S_{l+1}^{z}+L S_{l}^{z} S_{l+2}^{z}-h S_{l}^{z}\right],
$$

is a suitable starting point from which it is possible to develop the methodology. The spectrum consists of product states, $\left|\sigma_{1} \cdots \sigma_{N}\right\rangle$ with $\sigma_{l}=\uparrow, \downarrow$. The notation $\left|\sigma_{1} \cdots \sigma_{N}\right\rangle_{p}$ refers to sets of $p$ product states (of periodicity $p$ ) that transform into each other via translations.

The zero-temperature phase diagram at $h=0$ features three phases with periodicities $p=1,2,4$ (see figure 1). This includes phase $\Phi_{1}$ with nn spins aligned, phase $\Phi_{2}$ with nn spins anti-aligned, and phase $\Phi_{4}$ (at $L>\frac{1}{2}|J|$ ) with $n n$ spins alternatingly aligned and anti-aligned. The nnn spins are uniformly aligned in phases $\Phi_{1}, \Phi_{2}$ and uniformly anti-aligned in phase $\Phi_{4}$. At $h \neq 0$ and $J>0$, phases $\Phi_{2}$ and $\Phi_{4}$ persist, phase $\Phi_{1}$ is split up into two phases $\Phi_{1 \pm}$, and two new plateau phases $\Phi_{3 \pm}$ with periodicity $p=3$ are stabilized 1 The $h \neq 0$ phase diagram at $L=0$ features phases $\Phi_{2}$ in the sector $J>|h|$ and $\Phi_{1 \pm}$ at $h \gtrless 0$ in the other sectors.

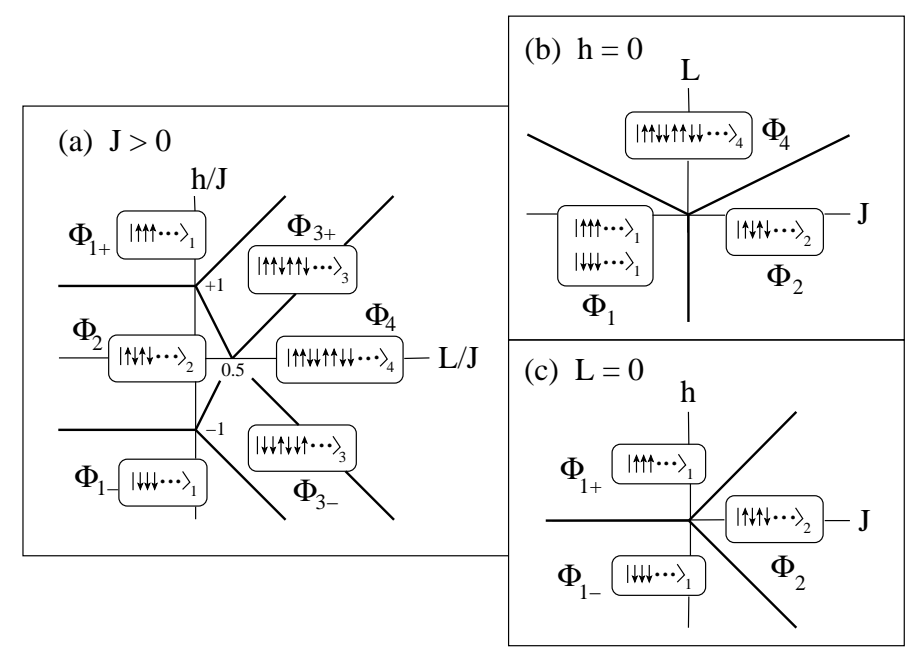

Figure 1. $T=0$ phase diagram (a) for positive nn coupling $J$ and nnn coupling $L$ of either sign in the parameter plane of scaled nnn-coupling $L / J$ and scaled magnetic field $h / J$, (b) for $h=0$ in the $(J, L)$ plane, and (c) for $L=0$ in the $(J, h)$-plane. The text refers to phases and regions in parameter space by the same names.

In section 2 we configure the physical vacua $\Phi_{1+}, \Phi_{2}$, and $\Phi_{4}$ as the pseudo-vacua of sets of statistically interacting particles. Salient features of the statistical mechanics of these particles are highlighted in section 3 (entropy, ordering tendencies) and section 4 (populations in competition). The emergence of structures from interactions on two levels is further discussed in section 5

\footnotetext{
${ }^{1}$ The $h \neq 0$ phase diagram is much simpler at $J<0$. Only three of the phases exist: $\Phi_{4}$ in the sector $L /|J|>|h / J|+1 / 2$ and $\Phi_{1 \pm}$ at $h \gtrless 0$ in the remaining sector of the $(L /|J|, h /|J|)$-plane.
} 


\section{Motifs, species, categories}

Motif is a term borrowed from music, literature, and visual arts, where it refers to fragments, themes, or patterns. Now well established in the natural sciences, it is used in biochemistry, for example, to describe patterns of nucleotides (codons) in DNA sequences. Motifs as a representation of particles in manybody systems are common in statistical mechanics [3, 7, 10]. In the present context, motifs are patterns of consecutive site variables $\sigma_{l}$ in Ising product states. Each motif characterizes either a particle of a particular species or an element of pseudo-vacuum. Our goal is to find motifs representing particles that have a definite energy irrespective of their location on the lattice relative to other particles. This requirement limits the number of ways particles can be identified. Every product eigenstate $\left|\sigma_{1} \cdots \sigma_{N}\right\rangle$ thus becomes a string of motifs.

Successive motifs interlink in a characteristic manner that depends on the range of interaction. (i) The $h$-term in Hamiltonian (1) has zero range. It permits the use of motifs that interlink by sharing no site variables (e.g. $\uparrow+\uparrow=\uparrow \uparrow$ ). All on-site energies are additive. (ii) The $J$-term has range one. It demands the use of motifs that interlink in one shared site variable (e.g. $\uparrow \bar{\uparrow}+\bar{\uparrow} \uparrow=\uparrow \bar{\uparrow} \uparrow$ ). This makes all nn-bond energies additive and the additivity of on-site energies can be maintained by specific rules. (iii) The $L$-term has range two, which requires that motifs interlink in two shared site variables (e.g. $\uparrow \bar{\uparrow} \bar{\uparrow}+\bar{\uparrow} \bar{\uparrow} \uparrow=\uparrow \bar{\uparrow} \bar{\uparrow} \uparrow$ ). This guarantees that all nnn-bond energies are additive without jeopardizing the additivity of nn-bond and on-site energies. Longer-range couplings demand a more extensive overlap of motifs and more elaborate rules for ensuring additivity of energy contributions from all Hamiltonian terms.

The search for motifs of a set of free particles that are excited from a given pseudo-vacuum is guided by the additional optimization criteria that aim for the fewest and shortest motifs. In the context of a spin1 Ising chain with nn-coupling we identified sets of six particles excited from twofold pseudo-vacua and sets of seven particles from non-degenerate pseudo-vacua [8]. Here we use the same strategy to identify three sets of particles that generate the full spectrum of (1) from pseudo-vacua $|\uparrow \uparrow \cdots\rangle_{1},|\uparrow \downarrow \uparrow \downarrow \cdots\rangle_{2}$, $|\uparrow \uparrow \downarrow \downarrow \uparrow \uparrow \downarrow \downarrow \cdots\rangle_{4}$, associated with the ground states at $h=0$.

The taxonomy of particles defined by their motifs involves structures as sorted into species and functions as emerging from categories. These features were the focus of reference [8]. In the present context the species will be very different but the categories will remain the same. We shall again encounter compacts, hosts, tags, hybrids, and no further categories. Compacts and hosts float in segments of pseudovacuum, tags are located inside hosts, and hybrids are tags with hosting capability. Particles from the same category but with different structures may collectively allow the emergence of new functions.

The number of product eigenstates that contain specific numbers $\left\{N_{m}\right\}$ of particles from all species of a given set is expressible by a multiplicity function $W\left(\left\{N_{m}\right\}\right)$. Its general structure, developed in the context of reference [8], remains operational without modification:

$$
\begin{aligned}
W\left(\left\{N_{m}\right\}\right) & =\frac{n_{\mathrm{pv}} N}{N-N^{(\alpha)}} \prod_{m=1}^{M}\left(\begin{array}{c}
d_{m}+N_{m}-1 \\
N_{m}
\end{array}\right), \quad N^{(\alpha)}=\sum_{m=1}^{M} \alpha_{m} N_{m}, \\
d_{m} & =A_{m}-\sum_{m^{\prime}=1}^{M} g_{m m^{\prime}}\left(N_{m^{\prime}}-\delta_{m m^{\prime}}\right),
\end{aligned}
$$

where $n_{\mathrm{pv}}$ is the multiplicity of the pseudo-vacuum, the $A_{m}$ are capacity constants, the $\alpha_{m}$ are size constants, and the $g_{\mathrm{mm}^{\prime}}$ are statistical interaction coefficients. The generalized Pauli principle proposed by Haldane [11] is encoded in [2b] with $d_{m}$ counting the number of open slots for particles of species $m$ in the presence of $N_{m^{\prime}}$ particles from any species $m^{\prime}$, thus encapsulating the essence of statistical interaction. All product states with particle content $\left\{N_{m}\right\}$ have energy

$$
E\left(\left\{N_{m}\right\}\right)=E_{\mathrm{pv}}+\sum_{m=1}^{M} N_{m} \epsilon_{m},
$$

where $\epsilon_{m}$ is the energy of particles from species $m$ relative to the pseudo-vacuum, which has (absolute) energy $E_{\mathrm{pv}}$. 


\subsection{Particles generated from $|\uparrow \downarrow \uparrow \downarrow \cdots\rangle_{2}$}

The physical vacuum in region $\Phi_{2}$ is the twofold Néel state $|\uparrow \downarrow \uparrow \cdots\rangle_{2}$, here selected as the pseudovacuum for $M=4$ species of particles with specifications as compiled in table 1 . The permissible configurations of particles from these species generate the complete spectrum of $\mathscr{H}$. The motifs interlink as illustrated in figure 2 Particles $m=3,4$ are hosts and particles $m=1,2$ tags. Hosts can be placed into segments of pseudo-vacuum and tags inside hosts. In this instance, hosts 3 accommodate tags 1 only and hosts 4 tags 2 only.

Table 1. Specifications of $M=4$ species of particles excited from the Néel state $\left(n_{\mathrm{pv}}=2\right)|\uparrow \downarrow \uparrow \cdots\rangle_{2}$ : motif, category, species, energy (relative to pseudo-vacuum), spin, capacity constants, size constants (left), and statistical interaction coefficients (right). Segments of $\ell$ vacuum elements, $\uparrow \downarrow \uparrow, \downarrow \uparrow \downarrow$, have energy $\ell(L-J) / 4$. At $h \neq 0$ the entries of $\epsilon_{m}$ must be amended by $-s_{m} h$.

\begin{tabular}{|ccc||cccc|}
\hline motif & category & $m$ & $\epsilon_{m}$ & $s_{m}$ & $A_{m}$ & $\alpha_{m}$ \\
\hline \hline$\uparrow \uparrow \uparrow$ & tag & 1 & $\frac{1}{2} J$ & $+\frac{1}{2}$ & 0 & 1 \\
$\downarrow \downarrow \downarrow$ & tag & 2 & $\frac{1}{2} J$ & $-\frac{1}{2}$ & 0 & 1 \\
$\downarrow \uparrow \uparrow \downarrow$ & host & 3 & $\frac{1}{2} J-L$ & $+\frac{1}{2}$ & $\frac{N-1}{2}$ & 1 \\
$\uparrow \downarrow \downarrow \uparrow$ & host & 4 & $\frac{1}{2} J-L$ & $-\frac{1}{2}$ & $\frac{N-1}{2}$ & 1 \\
\hline
\end{tabular}

\begin{tabular}{|c||rrrr|}
\hline$g_{m m^{\prime}}$ & 1 & 2 & 3 & 4 \\
\hline \hline 1 & 0 & 0 & -1 & 0 \\
2 & 0 & 0 & 0 & -1 \\
3 & $\frac{1}{2}$ & $\frac{1}{2}$ & $\frac{3}{2}$ & $\frac{1}{2}$ \\
4 & $\frac{1}{2}$ & $\frac{1}{2}$ & $\frac{1}{2}$ & $\frac{3}{2}$ \\
\hline
\end{tabular}

The energies of hosts and tags are calculated differently. In hosts (or elements of vacuum) we count interior $\mathrm{nn}$ bonds fully but the outermost $\mathrm{nn}$ bonds only half. In tags we do not count the nn bond on the left but do count the one on the right fully. All nnn bonds are counted fully in each particle (or element of vacuum). These rules are implemented in the entries for $\epsilon_{m}$. In analogous manner a spin $s_{m}$ can be assigned to particles from each species.

Tags have vanishing $A_{m}$ [8]. Open slots for tags are created by the placement of hosts. The number of tags that can latch on to a given host is only limited by the space to which the latter can expand in the process. In one product state tags (of one species) are lined up around the chain with no need of a host.

The $g_{m m^{\prime}}$ from table 1 as used in (2b) may be interpreted as follows: (i) Adding a tag does not change the capacity for further tags. Any slot taken by a tag opens up exactly one slot for a tag of the same kind. (ii) In the process of adding a tag its host must expand, which reduces the capacity for further hosts of either kind in the same manner. (iii) Adding a host increases the capacity for tags that it can host and has no effect on the capacity for tags it cannot host. (iv) Adding a host of any kind diminishes the capacity for further hosts of the same kind more strongly than for hosts of the other kind. Different hosts can be interlinked directly, whereas identical hosts must be spaced by at least one element of vacuum.

The two vectors of phase $\Phi_{2}$ are the pseudo-vacuum of the particles considered here. The two vectors of phase $\Phi_{1}$ are each a solid of $N$ close-packed tags of one kind. By contrast, the four vectors of phase $\Phi_{4}$ are each a solid of $\frac{1}{2} N$ hosts in an alternating pattern.

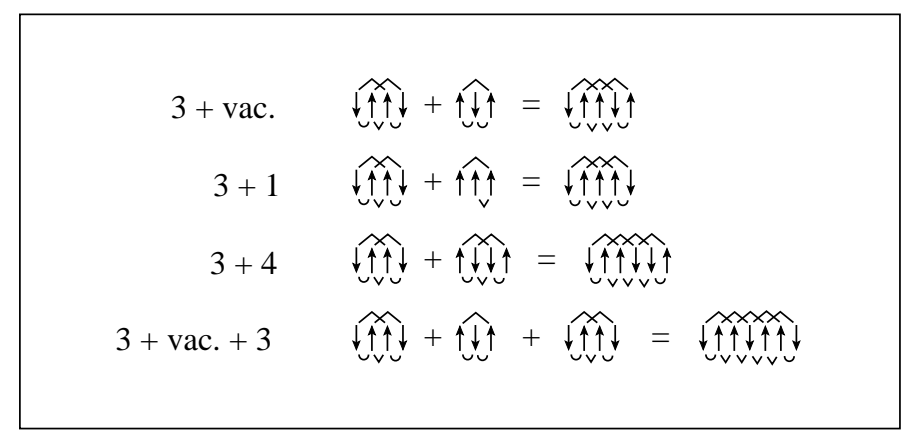

Figure 2. Interlinking elements of pseudo-vacuum and particles from table 1 Some $n n$ bonds $(U)$ are counted half, others $(\vee)$ full. All nnn bonds $(\wedge)$ are counted fully. 
For the case of vanishing nnn-coupling $(L=0)$ the four species of particles from table 1 can be replaced by the two species in table 2 . The motifs are shorter and interlink in one shared site variable. All nn bonds are now counted fully and all sites half in each motif. The new particles (named solitons) are well known from previous work [6, 12]. Their statistical interaction is semionic, an attribute shared with the spinons identified in the $X X Z$ and Haldane-Shastry models [13, 14]. In the taxonomy of reference [8] they are compacts. In figure 1(c) phase $\Phi_{2}$ is the pseudo-vacuum of solitons whereas phase $\Phi_{1+}\left(\Phi_{1-}\right)$ is a solid of $N$ spin-up (spin-down) solitons.

Table 2. Specifications of $M=2$ species of particles excited from the Néel state for $L=0\left(n_{\mathrm{pv}}=2\right)$ $|\uparrow \downarrow \uparrow \downarrow \cdots\rangle_{2}$. Segments of $\ell$ vacuum elements, $\uparrow \downarrow, \downarrow \uparrow$, have energy $-\ell J / 4$. At $h \neq 0$ the entries of $\epsilon_{m}$ must be amended by $-s_{m} h$.

\begin{tabular}{|ccc||cccc|}
\hline motif & cat. & $m$ & $\epsilon_{m}$ & $s_{m}$ & $A_{m}$ & $\alpha_{m}$ \\
\hline \hline$\uparrow \uparrow$ & comp. & + & $\frac{J}{2}$ & $+\frac{1}{2}$ & $\frac{N-1}{2}$ & 1 \\
$\downarrow \downarrow$ & comp. & - & $\frac{J}{2}$ & $-\frac{1}{2}$ & $\frac{N-1}{2}$ & 1 \\
\hline
\end{tabular}

\begin{tabular}{|c||cc|}
\hline$g_{m m^{\prime}}$ & + & - \\
\hline \hline+ & $\frac{1}{2}$ & $\frac{1}{2}$ \\
- & $\frac{1}{2}$ & $\frac{1}{2}$ \\
\hline
\end{tabular}

The motifs of solitons are not fragments of the original motifs. They interlink differently. At $L \neq 0$ we have two spin-up particles (tag 1 and host 3) and two spin-down particles (tag 2 and host 4). At $L=0$ the energies of both spin-up (or spin-down) particles become equal. In this case we can get away more economically with one spin-up and one spin-down soliton. Returning to $L \neq 0$, the energy of a soliton depends on its position relative to other solitons. The interaction energy is eliminated by switching back to the extended set of four species.

\subsection{Particles generated from $|\uparrow \uparrow \cdots\rangle_{1}$}

Phase $\Phi_{1}$ comprises the twofold spin-polarized state $|\uparrow \uparrow \uparrow \cdots\rangle_{1},|\downarrow \downarrow \downarrow \cdots\rangle_{1}$. Here we adopt the first vector as the pseudo-vacuum for $M=5$ species of particles with specifications compiled in table 3 . Three categories are represented. Host 2 accommodates only tag 4 whereas host 1 accommodates both tags and the hybrid. The hybrid, in turn, is capable of hosting both tags. The energies of hosts and tags are

Table 3. Specifications of $M=5$ species of particles excited from the spin-polarized state $\left(n_{\mathrm{pv}}=1\right)$ $|\uparrow \uparrow \cdots\rangle_{1}$. Segments of $\ell$ vacuum elements, $\uparrow \uparrow \uparrow$, have energy $\ell(L+J) / 4$. At $h \neq 0$ the entries of $\epsilon_{m}$ must be amended by $-s_{m} h$.

\begin{tabular}{|ccc||cccc|}
\hline motif & category & $m$ & $\epsilon_{m}$ & $s_{m}$ & $A_{m}$ & $\alpha_{m}$ \\
\hline \hline$\uparrow \uparrow \downarrow \downarrow \uparrow \uparrow$ & host & 1 & $-J-2 L$ & -2 & $N-3$ & 3 \\
$\uparrow \uparrow \downarrow \uparrow \uparrow$ & host & 2 & $-J-L$ & -1 & $N-2$ & 2 \\
$\downarrow \downarrow \downarrow$ & tag & 3 & 0 & -1 & 0 & 1 \\
$\uparrow \downarrow \uparrow \downarrow, \downarrow \uparrow \downarrow \uparrow$ & tag & 4 & $-J$ & -1 & 0 & 2 \\
$\downarrow \downarrow \uparrow \downarrow \downarrow$ & hybrid & 5 & $-J-L$ & -2 & 0 & 3 \\
\hline
\end{tabular}

\begin{tabular}{|c||rrrrr|}
\hline$g_{m m^{\prime}}$ & 1 & 2 & 3 & 4 & 5 \\
\hline \hline 1 & 4 & 3 & 1 & 2 & 3 \\
2 & 3 & 3 & 1 & 2 & 3 \\
3 & -1 & 0 & 0 & 0 & -1 \\
4 & -2 & -1 & 0 & 0 & -1 \\
5 & -1 & 0 & 0 & 0 & 0 \\
\hline
\end{tabular}


calculated as in section 2.1 The rules for hybrids are the same as those for tags. The pseudo-vacuum is spin-polarized and $s_{m}$ is not a spin in the usual sense. It enables us to write the magnetic-field contribution to $\epsilon_{m}$ in the form $-s_{m} h$.

Various combinations of hosts, tags, and hybrids are illustrated in figure 3. In the search for particles that are free of interaction energies, attention had to be paid to the requirement that the implantation of a tag or hybrid into a host (or a tag into hybrid) leaves the sums of aligned and anti-aligned nnn bonds invariant.

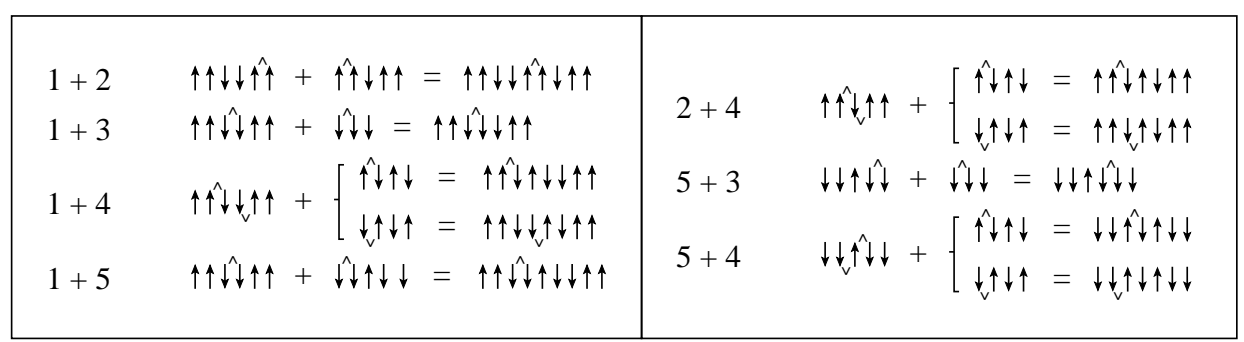

Figure 3. Interlinking particles from table 3 The number of nnn bonds is preserved and the number of $\mathrm{nn}$ bonds is reduced by one. The shared bond is marked by $\wedge$ or $\vee$.

The hosting capabilities of particles $m^{\prime}=1,2,5$ are encoded in negative statistical interaction coefficients $g_{m m^{\prime}}$. In all instances except one, we have $g_{m m^{\prime}}=-1$. The fact that host 1 has two interior slots to accommodate tag 4 requires that $g_{41}=-2$. Tag 3 has zero energy, a consequence of our choice of pseudovacuum. The physical vacuum in region $\Phi_{1}$ comprises the pseudo-vacuum $|\uparrow \uparrow \cdots\rangle_{1}$ and a solid of tags 3 , $|\downarrow \downarrow \cdots\rangle_{1}$. In region $\Phi_{2}$ the physical vacuum is a solid of negative-energy tags 4 (two vectors) and in region $\Phi_{4}$ a solid of negative-energy hosts 1 (four vectors).

For the case $L=0$ we can again get away with fewer particles that have shorter motifs and interlink with shorter overlap: one host and one tag as listed in table 4 In figure 1(c) phase $\Phi_{1+}$ is the pseudovacuum, phase $\Phi_{1-}$ is a solid of tags, and phase $\Phi_{2}$ is a solid of hosts. The two species of table 4 are free of $\mathrm{nn}$ interaction energies. The $\mathrm{nnn}$ interaction energies between them can be eliminated if we allow them to assemble into the five species of table 3 .

Table 4. Specifications of $M=2$ species of particles excited from the spin-polarized state for $L=0\left(n_{\mathrm{pv}}=1\right)$ $|\uparrow \uparrow \cdots\rangle_{1}$. Segments of $\ell$ vacuum elements, $\uparrow \uparrow$, have energy $\ell J / 4$. At $h \neq 0$ the entries of $\epsilon_{m}$ must be amended by $-s_{m} h$.

\begin{tabular}{|ccc||cccc|}
\hline motif & cat. & $m$ & $\epsilon_{m}$ & $s_{m}$ & $A_{m}$ & $\alpha_{m}$ \\
\hline \hline$\uparrow \downarrow \uparrow$ & host & $H$ & $-J$ & -1 & $N-1$ & 1 \\
$\downarrow \downarrow$ & tag & $T$ & 0 & -1 & 0 & 1 \\
\hline
\end{tabular}

\begin{tabular}{|c||rr|}
\hline$g_{m m^{\prime}}$ & $H$ & $T$ \\
\hline \hline$H$ & 2 & 1 \\
$T$ & -1 & 0 \\
\hline
\end{tabular}

\subsection{Particles generated from $|\uparrow \uparrow \downarrow \downarrow \uparrow \uparrow \cdots\rangle_{4}$}

In region $\Phi_{4}$ the fourfold state $|\uparrow \uparrow \downarrow \downarrow \uparrow \uparrow \cdots\rangle_{4}$ is the physical vacuum. Our search for free particles that generate the entire spectrum from this state configured as pseudo-vacuum has produced $M=4$ compacts with motifs and specifications compiled in table 5. All particles or elements of pseudo-vacuum comprise two $\mathrm{nn}$ bonds and one nnn bond. They again interlink by sharing one nn bond. Their energy content consists of one half of each nn bond energy plus the full nnn bond energy.

The assignment of a spin $\pm \frac{1}{2}$ to the four particles is based on the following reasoning. Take two interlinked elements of pseudo-vacuum with zero spin, $\uparrow \uparrow \downarrow \downarrow$ or $\downarrow \downarrow \uparrow \uparrow$, add one of the four particles, and check the spin of the resulting entity. For example, $\uparrow \uparrow \downarrow \downarrow+\uparrow \uparrow \uparrow \hat{=} \uparrow \uparrow \uparrow \downarrow \downarrow$ and $\uparrow \uparrow \downarrow \downarrow+\uparrow \downarrow \uparrow \hat{=} \uparrow \uparrow \downarrow \uparrow \downarrow$ produce entities with spin $+\frac{1}{2}$. 
Table 5. Specifications of $M=4$ species of particles excited from the state $\left(n_{\mathrm{pv}}=4\right)|\uparrow \uparrow \downarrow \downarrow \uparrow \uparrow \cdots\rangle_{4}$. Segments of $\ell$ vacuum elements $\uparrow \uparrow \downarrow, \uparrow \downarrow \downarrow, \downarrow \downarrow \uparrow, \downarrow \uparrow \uparrow$ have energy $-\ell L / 4$. At $h \neq 0$ the entries of $\epsilon_{m}$ must be amended by $-s_{m} h$.

\begin{tabular}{|ccc||cccc|}
\hline motif & category & $m$ & $\epsilon_{m}$ & $s_{m}$ & $A_{m}$ & $\alpha_{m}$ \\
\hline \hline$\uparrow \uparrow \uparrow$ & compact & 1 & $\frac{1}{4}(2 L+J)$ & $+\frac{1}{2}$ & $\frac{N-1}{4}$ & 1 \\
$\downarrow \downarrow \downarrow$ & compact & 2 & $\frac{1}{4}(2 L+J)$ & $-\frac{1}{2}$ & $\frac{N-1}{4}$ & 1 \\
$\uparrow \downarrow \uparrow$ & compact & 3 & $\frac{1}{4}(2 L-J)$ & $+\frac{1}{2}$ & $\frac{N+1}{4}$ & 1 \\
$\downarrow \uparrow \downarrow$ & compact & 4 & $\frac{1}{4}(2 L-J)$ & $-\frac{1}{2}$ & $\frac{N+1}{4}$ & 1 \\
\hline
\end{tabular}

\begin{tabular}{|c||rrrr|}
\hline$g_{m m^{\prime}}$ & 1 & 2 & 3 & 4 \\
\hline \hline 1 & $\frac{1}{4}$ & $\frac{1}{4}$ & $-\frac{1}{4}$ & $\frac{3}{4}$ \\
2 & $\frac{1}{4}$ & $\frac{1}{4}$ & $\frac{3}{4}$ & $-\frac{1}{4}$ \\
3 & $\frac{1}{4}$ & $\frac{1}{4}$ & $\frac{3}{4}$ & $-\frac{1}{4}$ \\
4 & $\frac{1}{4}$ & $\frac{1}{4}$ & $-\frac{1}{4}$ & $\frac{3}{4}$ \\
\hline
\end{tabular}

Four coefficients $g_{m^{\prime}}$ are negative. Here we do not have the standard host-tag scenario described previously. To understand the variant scenarios we note that each motif (four elements of vacuum and four particle species) can be followed only by two out of eight motifs. Only the motifs of particles 1,2 can follow themselves. Thus adding a particle $m^{\prime}=1$ or $m^{\prime}=2$ merely reduces the total number of open slots for further particles of any species. This explains that all coefficients in the first two columns are equal and positive. The coefficients in columns $m^{\prime}=3,4$ reflect stronger exclusion in some instances and accommodation in others, involving two distinct mechanisms.

(i) We note that particles 3,4 accommodate each other mutually. The addition of a particle $m^{\prime}=3$ opens up a slot for a particle $m=4$ and vice versa. This mutual accommodation is reflected in the coefficients $g_{34}=g_{43}=-1 / 4$. At the same time the addition of a particle $m^{\prime}=3$ or $m^{\prime}=4$ closes down a slot for further particles of the same species. This accounts for the more strongly positive coefficients $g_{33}=g_{44}=3 / 4$.

(ii) The sequence between any motif and the motif of particle $m=1$ can always be shortened by the insertion of a particle $m^{\prime}=3$ wherever it fits. By contrast, the insertion of a particle $m^{\prime}=4$ wherever it fits will lengthen that sequence. The presence of a particle $m^{\prime}=3$ thus increases the capacity of the system for particles $m=1$ and the presence of a particle $m^{\prime}=4$ has the opposite effect. This is reflected in the coefficients $g_{13}=-1 / 4, g_{14}=3 / 4$. Analogous reasoning explains the tabulated values of $g_{24}$ and $g_{23}$.

The ferromagnetic (FM) phase $\Phi_{1}$ consists of two states with broken spin-flip symmetry, one being a solid of particles 1 , the other a solid of particles 2 . The antiferromagnetic (AFM) phase $\Phi_{2}$ also consists of two states, each solid composed of particles 3,4 in an alternating sequence with broken translational symmetry. FM particles 1,2 and AFM particles 3,4 both have spin $s_{m}= \pm \frac{1}{2}$. By interlinking differently, the former produce a uniform magnetization in phase $\Phi_{1}$ and the latter a staggered magnetization in phase $\Phi_{2}$.

\section{Entropy landscapes}

The statistically interacting particles from the three sets identified in section 2 have definite shapes and energies. These floating objects are assembled from localized spins by the $n n$ and nnn couplings of Hamiltonian (1). The Ising chain is an open system of particles with energies $\epsilon_{m}$ depending on $J, L, h$. Here we abandon the Ising context and focus entirely on particles with interlinking motifs.

The statistical interaction between the particles depends on their shapes and on the nature of the pseudo-vacuum, but not on the particle energies $\epsilon_{m}$. It is instructive to explore the effects of statistical interactions produced by particular shapes in a setting where particle energies do not factor in. To this end we consider the configurational entropy as derived from the multiplicity expression (2) for $N, N_{m} \gg$ 1 via $S=k_{\mathrm{B}} \ln W$ :

$$
S\left(\left\{N_{m}\right\}\right)=k_{\mathrm{B}} \sum_{m=1}^{M}\left[\left(N_{m}+Y_{m}\right) \ln \left(N_{m}+Y_{m}\right)-N_{m} \ln N_{m}-Y_{m} \ln Y_{m}\right],
$$




$$
Y_{m} \doteq A_{m}-\sum_{m^{\prime}=1}^{M} g_{m m^{\prime}} N_{m^{\prime}} .
$$

The functional dependence of $S$ on the populations $N_{m}$ of particle species from a given set determines an entropy landscape shaped by the statistical interactions alone.

If only one species is present, the statistical interaction reduces to an exclusion principle. The function $S\left(N_{m}\right)$ vanishes identically for any species of tags or hybrids, which have $A_{m}=0$ and $g_{m m}=0$. These particles can only exist inside hosts. Compacts and hosts exist in segments of pseudo-vacuum. They have $A_{m} \propto N$ and $g_{m m}>0$. The entropy $S\left(N_{m}\right)$ of a single species of hosts or compacts is nonzero for $0<$ $N_{m}<A_{m} / g_{m m}$ and zero at the endpoints.

The signature of the statistical interaction between any two species $m, m^{\prime}$ is best visualized in a reduced entropy landscape, in contour plots of the entropy per site, $\bar{S}\left(\bar{N}_{m}, \bar{N}_{m^{\prime}}\right)=S\left(N_{m} / N, N_{m^{\prime}} / N\right) / N$ with the populations of all other species suppressed. All thermodynamic processes described in the following are understood to be quasi-static and to be implemented in an open system 2 Equilibration would be problematic in closed, one-dimensional systems of particles from more than one species.

\subsection{Hosts, tags, hybrids}

Beginning with the four species from section 2.1 we consider the entropy landscapes pertaining to the two hosts, a host and the tag it does or does not accommodate, and the two tags. In each instance we vary the population densities of two species over the permissible range while keeping the population densities of the other two species constant at very low values.

The entropy landscape of the two hosts as shown in figure 4 (a) has borders of quadrilateral shape. The four corners correspond to pure phases with vanishing entropy. Hosts interlink with elements of pseudo-vacuum in multiple configurations. This explains the nonzero entropy along the two sides on the axes. The other two sides represent states that are crowded with hosts of both species. If $\bar{N}_{3}=\bar{N}_{4}$ the state of maximum population density is unique. Hosts 3,4 interlink directly. Hosts 3,3 or 4,4 are spaced by at least one element of pseudo-vacuum. The corner states $\Phi_{3 \pm}$ contain $\frac{1}{3} N$ close-packed hosts from a single species. The state $\Phi_{4}$ contains $\frac{1}{2} N$ close-packed hosts from both species arrayed in an alternating sequence. Heading from point $\Phi_{3+}$ toward point $\Phi_{4}$ involves the repeated replacement of one host 3 by three hosts 4.

A qualitatively different entropy landscape pertains to host 3 and tag 1 (hosted by 3 ) as shown in figure 4 (b). The borders are of triangular shape. Hosts 3 alone (vertical leg) generate entropy but tags 1 alone (horizontal leg) do not. All tags 1 are arrayed uniformly inside hosts 3. Only the expanded hosts of multiple sizes have positional disorder, not the tags inside. As the hosts disappear, so does the entropy. The tags contribute to the entropy only indirectly by expanding hosts to different sizes. Close-packed configurations of hosts stuffed with tags are represented by points on the hypotenuse. Near the middle the entropy is largest, generated by close-packed hosts expanded to many different sizes. Near one end, we have many more hosts but with only few small sizes represented. Near the other end, we have few hosts expanded to large sizes. Either trend reduces the entropy.

The entropy landscape is yet different for host 4 and tag 1 (not hosted by 4) as shown in figure 4 (c). All tags 1 are confined to very few hosts 3, incapable of producing any significant entropy by themselves (horizontal leg). Almost all hosts 4 have the minimal size due to the near absence of tags 2 . These hosts 4 produce positional entropy along the vertical leg. The hypotenuse describes configurations of near uniform segments of hosts 4 separated by rare hosts 3 filled to various sizes with uniform arrays of tags 1 . This arrangement has very low entropy. Starting with any density of hosts 4, the entropy always decreases when we add tags 1 . All tags 1 added replace elements of pseudo-vacuum and thus reduce the options for positioning hosts 4 . The tags themselves are lumped together inside few hosts 3.

The two tags 1 and 2 have an entropy landscape (not shown) that is also triangular but very flat near zero entropy. The two species of tags do not mix. They are arrayed uniformly in separate hosts, present only in very low densities.

The entropy landscapes of the five species of hosts, tags, and hybrids from section 2.2 are all of triangular shape. The hybrid 5 gives rise to one new feature, e.g. in combination with tag 3 as illustrated in

\footnotetext{
${ }^{2}$ The Ising model [1] is one particular realization with specific particle energies $\epsilon_{m}$.
} 

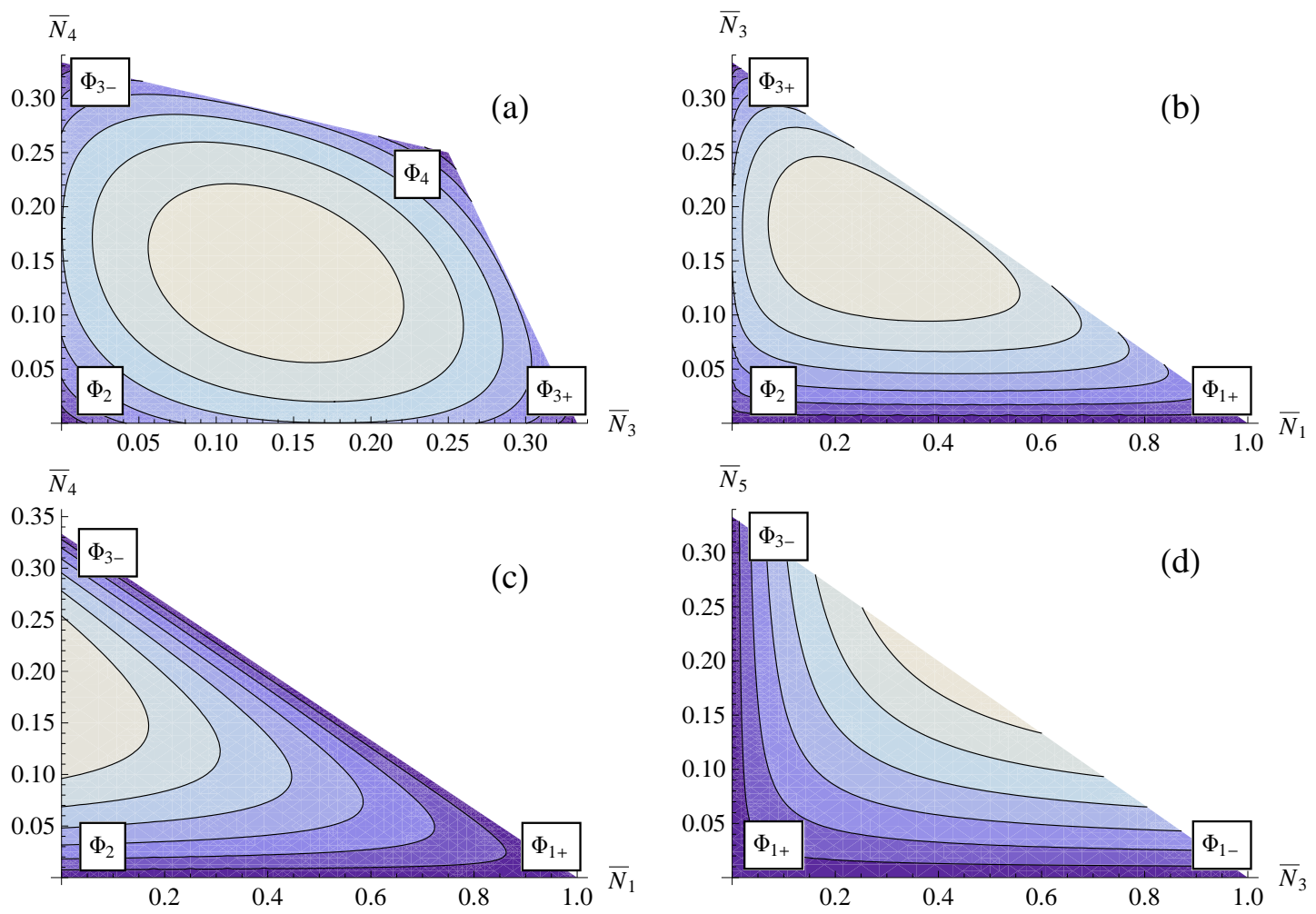

Figure 4. (Color online) Entropy per site, $\bar{S} / k_{\mathrm{B}}$, versus population densities, $\bar{N}_{m}, \bar{N}_{m^{\prime}}$, of two species from (a)-(c) table 1 and (d) from table 3 The population densities of the other species are kept at $10^{-5}$. The contours are at (a) $0.071 \ell$, (b) $0.068 \ell$, (c) $0.039 \ell$, (d) $0.058 \ell, \ell=1, \ldots, 6$.

figure 4(d). Both species exist inside hosts 1 or 2. Tags 3 alone or hybrids 5 alone produce no significant entropy for reasons already stated. However, the two species can coexist inside the same host in many different configurations. Almost all entropy is now generated inside the few hosts available.

In surveying the four panels of figure 4 we see that the entropy maximum is realized under diverse circumstances, with both species or only one species present at intermediate density, or with two species in close-packed configurations. As expected, maximum positional ordering of the particles (zero entropy) occurs if no particles are present or if the system is close-packed with one species. Close-packing with two species is also found to produce maximum positional order either through alternate stacking [panel (a)] or through segregation [panel (c)].

\subsection{Compacts}

All four species of compacts from section 2.3 interlink with elements of pseudo-vacuum but only selectively with themselves or each other. This produces two features in their entropy landscapes not seen in the previous cases. Compacts 1 and 2, which interlink with themselves but not with each other or with any other compact, generate a triangular entropy landscape as shown in figure 5(a). The entropy is nonzero along each leg, where compacts from one species mix with elements of pseudo-vacuum. The entropy is zero along the hypotenuse, where compacts are close-packed. The system has the highest capacity for compacts 1 and 2 if they are segregated.

The most remarkable entropy landscape pertains to compacts 3 and 4 as shown in figure 5(b). These two compacts interlink with each other but not with themselves or with any other compact. The border is a quadrilateral as already seen in figure 4(a) but with the sides away from the axes now slanted positively. If we start filling the system with compacts 3, the entropy first increases from zero and then decreases again down to zero when capacity is reached at $N_{3}=\frac{1}{3} N$ in a uniformly stacked array. Interestingly, we 

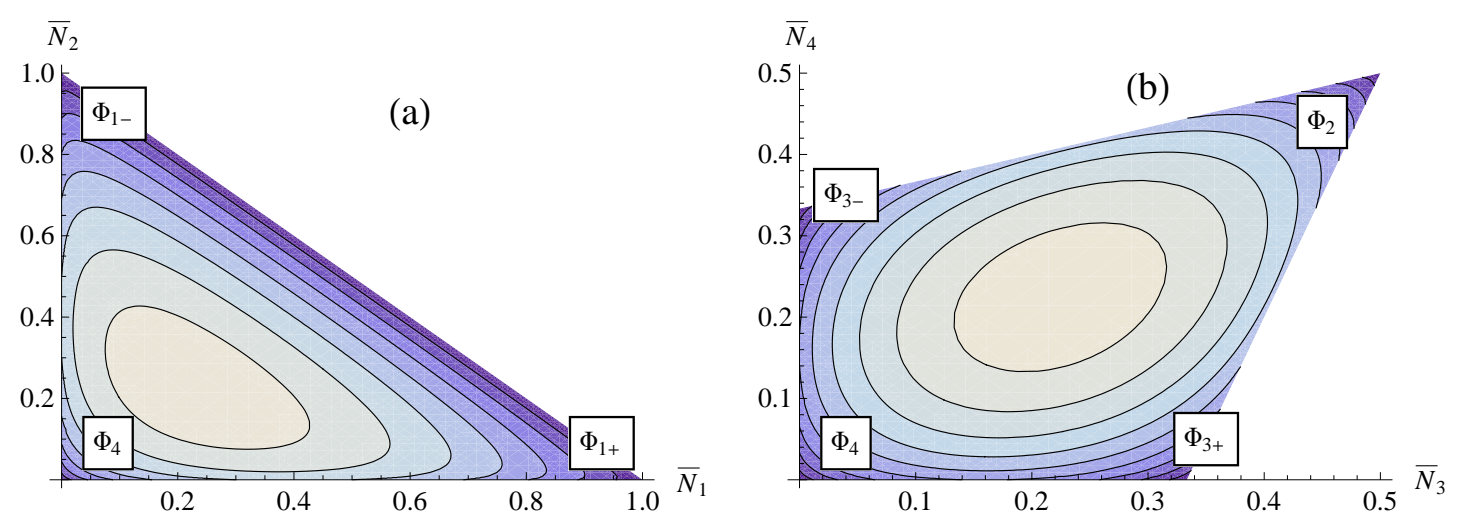

Figure 5. (Color online) Entropy per site, $\bar{S} / k_{\mathrm{B}}$, versus population densities, $\bar{N}_{m}, \bar{N}_{m^{\prime}}$, of two species from table 5 The population densities of the other species are kept at $10^{-5}$. The contours are at (a) $0.070 \ell$, (b) $0.071 \ell, \ell=1, \ldots, 6$.

do not have to remove any compacts 3 to make space for compacts 4 . In fact, for every three compacts 4 added we can add one more compact 3 . The entropy rises in the process and then returns to zero when the numbers of both species have become equal and reached the value $\frac{1}{2} N$.

Consider a macroscopic system initially in the pseudo-vacuum of compacts. What happens to the entropy if we add equal numbers of particles from two of the four species until capacity is reached? The answer is shown in figure 6. At low density, the positional disorder is little affected by the different shapes of the particles. The curves overlap close to perfectly. At higher densities the different shapes dictate the presence or absence of ordering tendencies and the types of ordering realized. Particles 1,1 or 2,2 or 3,4 interlink directly. Two close-packed particles 1,2 or 3,3 or 4,4 have two vacuum elements in a particular sequence between them.

With increasing (averaged) densities $\bar{N}_{1}=\bar{N}_{2}$ or $\bar{N}_{3}=\bar{N}_{4}$ the entropy varies along the same curve even though the associated equilibrium states are very different. In the case of particles 1,2 the shapes favor single-species clustering when the space becomes crowded. Mixed-species clustering is favored in the case of particles 3,4 . When capacity is reached, the entropy has returned to zero. The equilibrium state is then fully phase separated in one case, consisting of two equal-size single-species clusters, or homogeneous in the other case, consisting of one mixed-species cluster.

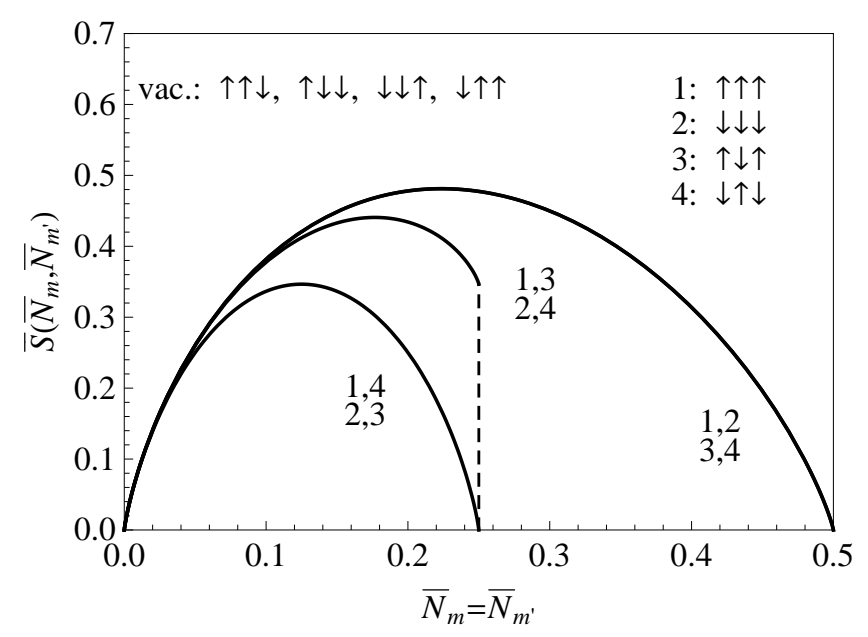

Figure 6. Entropy $\bar{S} / k_{\mathrm{B}}$ per lattice site as function of the density $\bar{N}_{m}=\bar{N}_{m^{\prime}}$ of two species of compacts from table 5 
Particles 1,4 (or 2,3) are close-packed with three vacuum elements between them. By contrast, closepacked particles 1,1 interlink directly and close-packed particles 4,4 have two vacuum elements between them. With increasing (averaged) densities $\bar{N}_{1}=\bar{N}_{4}$, single-species clustering crowds out mixed-species clustering. Capacity is reached earlier. The entropy returns to zero as before. The equilibrium is fully phase-separated. The two clusters are of unequal size.

Particles 1,3 (or 2,4) are separated by only one vacuum element when close-packed. The consequence is that with increasing (averaged) densities $\bar{N}_{1}=\bar{N}_{3}$ single-species and mixed-species clustering are equally favorable (compared to loose particles). The system reaches capacity in an amorphous state. The entropy stays nonzero. The higher rise of the entropy compared to the previous case is explained by the smaller size of close-packed mixed-species pairs, which increases the positional disorder at equilibrium.

\section{Statistical mechanics}

The statistical mechanical analysis of the particles with shapes identified in section 2 can be performed for open or closed systems. Here we consider an open system. Wu's analysis [15] for a generic situation starts from the expression

$$
Z=\sum_{\left\{N_{m}\right\}} W\left(\left\{N_{m}\right\}\right) \exp \left(-\sum_{m} \frac{\epsilon_{m} N_{m}}{k_{\mathrm{B}} T}\right)
$$

for the grandcanonical partition function, where $\epsilon_{m}$ are the particle energies and $W\left(\left\{N_{m}\right\}\right)$ the multiplicity function 3 That analysis produces the general result

$$
Z=\prod_{m}\left(\frac{1+w_{m}}{w_{m}}\right)^{A_{m}},
$$

where the (real, positive) $w_{m}$ are the solutions of the coupled nonlinear algebraic equations,

$$
\frac{\epsilon_{m}}{k_{\mathrm{B}} T}=\ln \left(1+w_{m}\right)-\sum_{m^{\prime}} g_{m^{\prime} m} \ln \left(\frac{1+w_{m^{\prime}}}{w_{m^{\prime}}}\right) .
$$

The capacity constants $A_{m}$, and the mutual interaction coefficients $g_{m m^{\prime}}$ are tabulated in section 2 Arbitrary energies $\epsilon_{m}$ can be assigned to each particle species. With the $w_{m}$ from (7), the average numbers of particles can be derived from (5) via

$$
\left\langle N_{m}\right\rangle=-k_{\mathrm{B}} T \frac{\partial \ln Z}{\partial \epsilon_{m}},
$$

which, when carried out using (6) and (7), leads to the linear coupled equations 4

$$
w_{m}\left\langle N_{m}\right\rangle+\sum_{m^{\prime}} g_{m m^{\prime}}\left\langle N_{m^{\prime}}\right\rangle=A_{m} .
$$

In similar fashion we can derive from (6) and (7) correlations between particle populations, specifically the covariances

$$
\left\langle\left\langle N_{m} N_{m^{\prime}}\right\rangle\right\rangle \doteq\left\langle N_{m} N_{m^{\prime}}\right\rangle-\left\langle N_{m}\right\rangle\left\langle N_{m^{\prime}}\right\rangle=\left(k_{\mathrm{B}} T\right)^{2} \frac{\partial^{2} \ln Z}{\partial \epsilon_{m} \partial \epsilon_{m^{\prime}}} .
$$

The entropy inferred from (6) can be expressed as a function of the $\left\langle N_{m}\right\rangle$ alone, namely by the function $S\left(\left\{\left\langle N_{m}\right\rangle\right\}\right)$ from (4).

It is instructive to compare this method with the transfer matrix method [16] in the context of the Ising chain (1). The latter operates with coupled degrees of freedom of minimal structure whereas the former operates with degrees of freedom that are no longer coupled but have more complex structures. Ising spins are tied to lattice sites whereas particles are floating. The number $N$ of Ising spins is fixed whereas the numbers $N_{m}$ of particles from each species are fluctuating. Ising spins at different sites are

\footnotetext{
${ }^{3}$ The chemical potential is zero for all species, a consequence of the energy scales chosen in section 2

${ }^{4}$ The analysis assumes that $N \gg 1$ and $\left\langle N_{m}\right\rangle \gg 1$. Any part of a nonzero $A_{m}$ that is of O(1) is irrelevant. Wu's derivation of [6] proceeds via [9]. The calculation of [9] from [8] via [6] and (7) is merely a check of internal consistency.
} 
distinguishable whereas particles from the same species are not. The canonical partition function $Z_{N}$ from the transfer matrix analysis is related to the grand partition function (6) via

$$
Z=\mathrm{e}^{E_{\mathrm{pv}} / k_{\mathrm{B}} T} Z_{N},
$$

where $E_{\mathrm{pv}}$ is the energy of the pseudo-vacuum in use.

For a brief demonstration of how particles with interlinking motifs and different energies compete for space in the presence of thermal fluctuations and produce long-range ordering tendencies at low $T$ as a consequence, we assign the energies of (1) at $h=0$ as tabulated. All thermodynamic quantities of interest can then conveniently be expressed in terms of the function 5

$$
u\left(K_{J}, K_{L}\right)=\cosh K_{J}+\sqrt{\sinh ^{2} K_{J}+\mathrm{e}^{4 K_{L}}}, \quad K_{J} \doteq \frac{J}{4 k_{\mathrm{B}} T}, \quad K_{L} \doteq \frac{L}{4 k_{\mathrm{B}} T} .
$$

The familiar transfer matrix result then reads [16

$$
Z_{N}=\left[u\left(K_{J}, K_{L}\right) \mathrm{e}^{-K_{L}}\right]^{N}
$$

\subsection{Analysis from $|\uparrow \downarrow \uparrow \downarrow \cdots\rangle_{2}$}

The physically relevant solution of equations (7) for the four species of hosts and tags introduced in section 2.1 is

$$
w_{1}=w_{2}=u\left(K_{J}, K_{L}\right) \mathrm{e}^{K_{J}}-1, \quad w_{3}=w_{4}=w_{1} \mathrm{e}^{-4 K_{L}} .
$$

The grand partition function (6) becomes

$$
Z=\left[u\left(K_{J}, K_{L}\right) \mathrm{e}^{-K_{J}}\right]^{N},
$$

consistent with (13) via (11). Equations (9) yield

$$
\left\langle\bar{N}_{1}\right\rangle=\left\langle\bar{N}_{2}\right\rangle=\left\langle\bar{N}_{3}\right\rangle \frac{1}{w_{1}}, \quad\left\langle\bar{N}_{3}\right\rangle=\left\langle\bar{N}_{4}\right\rangle=\frac{1}{2} \frac{w_{1}}{w_{1} w_{3}+2 w_{1}+1} .
$$

In figure 7 we show contour plots of the population densities of tags $m=1,2(\uparrow \uparrow \uparrow, \downarrow \downarrow \downarrow)$ and hosts $m=3,4(\downarrow \uparrow \uparrow \downarrow, \uparrow \downarrow \downarrow \uparrow)$. Lowering $T$ at fixed $J, L$ means moving from the center radially outward in a given direction. Any particular population density either increases or decreases from the common value $\left\langle\bar{N}_{m}\right\rangle=\frac{1}{8}$ at $T=\infty$. In region $\Phi_{2}$ all $\left\langle\bar{N}_{m}\right\rangle$ decrease and reach zero at $T=0$. All particle energies $\epsilon_{m}$ are positive. Throughout region $\Phi_{4}$ the host energies are negative. The host population densities $\left\langle\bar{N}_{3}\right\rangle=\left\langle\bar{N}_{4}\right\rangle$ increase as $T$ is lowered and reach the value $\frac{1}{4}$ at $T=0$. In part of this region, at $J<0$, the tags have negative energies as well, but their energy density is less negative than that of hosts. Hence tags are crowded out by hosts. In region $\Phi_{1}$ the host population diminishes and the tag population proliferates toward $\left\langle\bar{N}_{1}\right\rangle=\left\langle\bar{N}_{2}\right\rangle=\frac{1}{2}$ as $T$ is lowered. In part of this region, for $L>0$, the hosts have lower (negative) excitation energies than the tags. Nevertheless, the hosts are crowded out by the tags due to their larger size.

On the $\Phi_{1}-\Phi_{2}$ boundary $(J=0, L<0)$ the hosts have $\epsilon_{m}>0$ and the tags $\epsilon_{m}=0$. As $T$ is lowered the host population decreases, leaving more room for tags and elements of pseudo-vacuum, whose motifs do not interlink directly. At low $T$ the few remaining hosts act as surfactants between segments of tags inside and segments of pseudo-vacuum outside [8]. The ground state is fourfold degenerate. Two vectors contain no particles. The other two are solids formed by tags of one or the other kind. The density of tags averaged over the four vectors is $\left\langle\bar{N}_{1}\right\rangle=\left\langle\bar{N}_{2}\right\rangle=\frac{1}{4}$.

On the $\Phi_{2}-\Phi_{4}$ boundary ( $L=J / 2>0$ ) the particles with $\epsilon_{m}>0$ are tags and the particles with $\epsilon_{m}=0$ are hosts - a switch with drastic consequences. As $T$ is lowered the tag population decreases, surrendering the lattice to hosts and elements of pseudo-vacuum, whose motifs do interlink directly. A highly

\footnotetext{
${ }^{5}$ The mapping which relates the special cases $h=0$ and $L=0$ of Hamiltonian 1 with open boundary conditions by simple parameter transcription $J \leftrightarrow-h, L \leftrightarrow J[20]$ is evident in the partition function but has no impact on what is demonstrated here.
} 

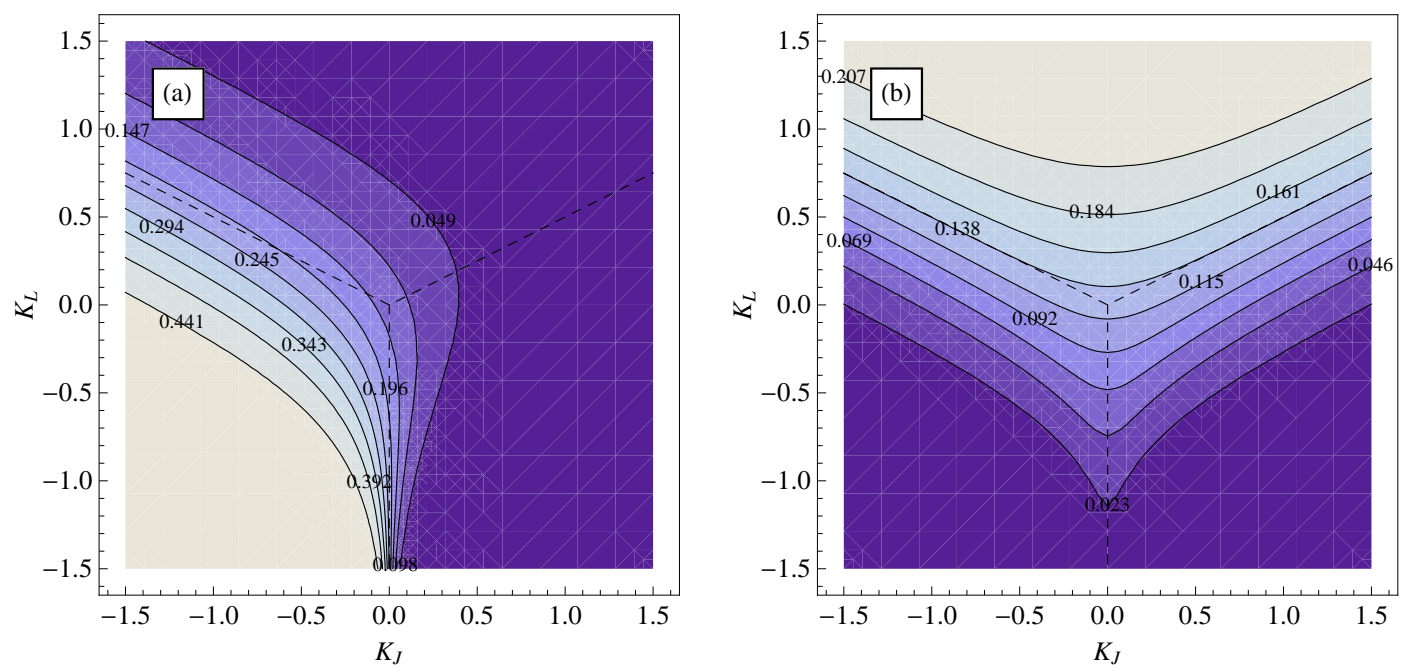

Figure 7. (Color online) Average number (per site) (a) $\left\langle\bar{N}_{1}\right\rangle=\left\langle\bar{N}_{2}\right\rangle$ of tags and (b) $\left\langle\bar{N}_{3}\right\rangle=\left\langle\bar{N}_{4}\right\rangle$ of hosts versus $K_{J}, K_{L}$. Plot (a) also represents the population density of FM particles discussed in section 4.2 The population density of AFM particles from that same set is the left-right mirror image of plot (a). The dashed lines indicate the $T=0$ phase boundaries of figure 1 (b).

degenerate ground state ensues. The limiting host population densities $(\underline{16})$ are $\left\langle\bar{N}_{3}\right\rangle=\left\langle\bar{N}_{4}\right\rangle=(5+\sqrt{5})^{-1} \simeq$ 0.138 .

Finally, on the $\Phi_{1}-\Phi_{4}$ boundary ( $L=-J / 2>0$ ), all particles have $\epsilon_{m}<0$. Hosts and tags have equal negative energy densities. All configurations of hosts and tags covering the lattice solidly have the same energy. The resultant entropy is the same as that along the $\Phi_{2}-\Phi_{4}$ boundary. The host population density remains the same, $\left\langle\bar{N}_{3}\right\rangle=\left\langle\bar{N}_{4}\right\rangle=(5+\sqrt{5})^{-1} \simeq 0.138$. The tag population density, calculated from (16), is $\left\langle\bar{N}_{1}\right\rangle=\left\langle\bar{N}_{2}\right\rangle=(2 \sqrt{5})^{-1} \simeq 0.224$.

\subsection{Analysis from $|\uparrow \uparrow \downarrow \downarrow \uparrow \uparrow \cdots\rangle_{4}$}

The grand partition function for the four compacts introduced in section 2.3becomes

$$
Z=\left[u\left(K_{J}, K_{L}\right) \mathrm{e}^{-2 K_{L}}\right]^{N},
$$

again consistent with (13) via (11), and where

$$
w_{1}=w_{2}=u\left(K_{J}, K_{L}\right) \mathrm{e}^{K_{J}}-1, \quad w_{3}=w_{4}=\left(1+w_{1}\right) \mathrm{e}^{-2 K_{J}}-1,
$$

are the solutions of equations (7), and equations (9) yield

$$
\left\langle\bar{N}_{1}\right\rangle=\left\langle\bar{N}_{2}\right\rangle=\frac{1}{2} \frac{w_{3}}{2 w_{1} w_{3}+w_{1}+w_{3}}, \quad\left\langle\bar{N}_{3}\right\rangle=\left\langle\bar{N}_{4}\right\rangle=\frac{1}{2} \frac{w_{1}}{2 w_{1} w_{3}+w_{1}+w_{3}} .
$$

The population densities of the FM particles $1,2(\uparrow \uparrow \uparrow, \downarrow \downarrow \downarrow)$ and the AFM particles $3,4(\uparrow \downarrow \uparrow, \downarrow \uparrow \downarrow)$ have a mirror-image relationship (figure $7{ }^{6}$ Lowering $T$ from infinity either leads to a depletion of FM particles (region $\Phi_{2}$ ) or to a depletion of AFM particles (region $\Phi_{1}$ ) or to a depletion of both kinds of particles (region $\Phi_{4}$ ).

If $T$ is lowered on the $\Phi_{1}-\Phi_{2}$ border, all four particles have equal and negative energies. What matters for the nature of the ground state is that FM particles and AFM particles interlink among themselves (albeit in different manner) but not with each other. The ground state thus consists of the four states that are either packed with FM particles or with AFM particles.

\footnotetext{
${ }^{6}$ Tag 2 from table 1 and FM compact 2 from table 5 have identical motifs and, therefore, identical (absolute) energies. However, they are different particles existing in different worlds. Nevertheless, their population densities are identical at $h=0$.
} 
Along the $\Phi_{1}-\Phi_{4}$ border, FM particles have zero energy and AFM particles have positive energies. The lowest energy level is highly degenerate, comprising states populated by FM particles in all permissible configurations. The average population density of these compacts is $\left\langle\bar{N}_{1}\right\rangle=\left\langle\bar{N}_{2}\right\rangle=(2 \sqrt{5})^{-1} \simeq 0.224$. The role of the AFM particles along the $\Phi_{2}-\Phi_{4}$ border is the same as that of the FM particles along the $\Phi_{1}-\Phi_{4}$ border in all respects except the way the motifs interlink.

\subsection{Analysis from $|\uparrow \uparrow \cdots\rangle_{1}$}

Equations (7) of the five species of hosts, tags, and hybrids introduced in section 2.2 have the solutions

$$
\begin{aligned}
& w_{3}=u\left(K_{J}, K_{L}\right) \mathrm{e}^{K_{J}}-1, \quad \frac{1+w_{4}}{\left(1+w_{3}\right)^{2}}=\mathrm{e}^{-4 K_{J}}, \quad \frac{1+w_{5}}{w_{3} w_{4}}=\mathrm{e}^{-4 K_{L}}, \\
& w_{1}=\frac{w_{4} w_{5}}{1+w_{4}} \mathrm{e}^{-4 K_{L}}, \quad w_{2}=\frac{1+w_{1}}{w_{1} w_{3}-1},
\end{aligned}
$$

implying

$$
Z=\left[u\left(K_{J}, K_{L}\right) \mathrm{e}^{K_{J}}\right]^{N}
$$

The solutions of equations (9) are again straightforward but, in this instance, unwieldy. Symmetries dictate that $\left\langle\bar{N}_{2}\right\rangle=\left\langle\bar{N}_{5}\right\rangle$. The population densities as presented graphically in figure 8 are more complex. Two of the species play only minor parts in the statistical mechanics. Hosts $2(\uparrow \uparrow \downarrow \uparrow \uparrow)$ and hybrids $5(\downarrow \downarrow \uparrow \downarrow \downarrow)$ are not abundantly present for any combination of coupling constants or any temperature. They barely make it to an average of five particles per hundred lattice sites under the most favorable equilibrium circumstances, whereas hosts $1(\uparrow \uparrow \downarrow \downarrow \uparrow \uparrow)$ make it to an average of up to 25 and the tags $3(\downarrow \downarrow \downarrow), 4(\uparrow \downarrow \uparrow \downarrow$ $, \downarrow \uparrow \downarrow \uparrow)$ to averages of up to 50 .

At $L>|J| / 2$, host 1 has the lowest energy of all by a considerable margin. Its population density is dominant in that region. At $J>0, L<0$ the equilibrium state is dominated by tags 4 and at $J<0, L<0$ by tags 3. In both regions these low-energy tags can only exist as attachments to higher-energy hosts. As $T$ is lowered fewer and fewer hosts have more and more tags attached to them. At $T=0$ the last host particle is replaced by a low-energy tag in a configuration where each tag is attached to its neighbor on the left around the chain.

In the remaining two regions at $0<L<|J| / 2$ two or three species of particles are in competition for dominance with energy, size, and category all factoring in. The smallest size among the low-energy particles happens to win in both regions as the temperature is turned down: tag 3 at $J<0$ and tag 4 at $J>0$. There is no region where the total particle population is thinned out entirely as $T \rightarrow 0$. Tags 3 have zero energy. They only disappear if they are crowded out by particles with negative energy. Our choice of a pseudo-vacuum that does not coincide with the physical vacuum.

Along the $\Phi_{1}-\Phi_{2}$ border tags 3,4 both have zero energy while all other particles have positive energies. Tags 3 do not interlink with tags 4 . The ground-state degeneracy remains low (fourfold). On the $\Phi_{1}-\Phi_{4}$ border we have again two zero-energy species (hosts 1 and tags 3) and three positive-energy species. However, the former two do interlink. The ground-state degeneracy is high. The population densities are $\left\langle\bar{N}_{1}\right\rangle=(5+\sqrt{5})^{-1} \simeq 0.138$ for the hosts and $\left\langle\bar{N}_{3}\right\rangle=(2 \sqrt{5})^{-1} \simeq 0.224$ for the tags.

A considerably more complex scenario unfolds on the $\Phi_{2}-\Phi_{4}$ border. Four species of particles of three different sizes and with three different negative energies are in competition. Only tag 3 (with zero energy) stands by. Of the four competing particles, 1,2,5 can host and 4,5 can be hosted. Hosts 2 and hybrids 5 are absent at $T=0$ except on the phase boundary. There we have $\left\langle\bar{N}_{1}\right\rangle=\left\langle\bar{N}_{4}\right\rangle=\frac{1}{9},\left\langle\bar{N}_{2}\right\rangle=\left\langle\bar{N}_{5}\right\rangle=\frac{1}{18}$. At $T>0$ the particles 2,5 maintain significant populations only near the phase boundary.

\subsection{Entropy}

The three sets of particles produce distinct entropy landscapes in the space of population densities as discussed in section 3 This diversity is attributable to the different structures of the particle species and their mutual statistical interactions as encapsulated in the entropy expression (4). However, when applied to a particular physical context, where all particle energies $\epsilon_{m}$ are functions of Hamiltonian parameters, 
the function $S\left(\left\{\left\langle N_{m}\right\rangle\right\}\right)$ inferred from $\left(4_{4}^{7} 7\right.$ encodes, at any given temperature, the same entropy landscape for all three sets of particles in the space of these parameters. That landscape is shown in figure 8 (d) for the situation at hand.
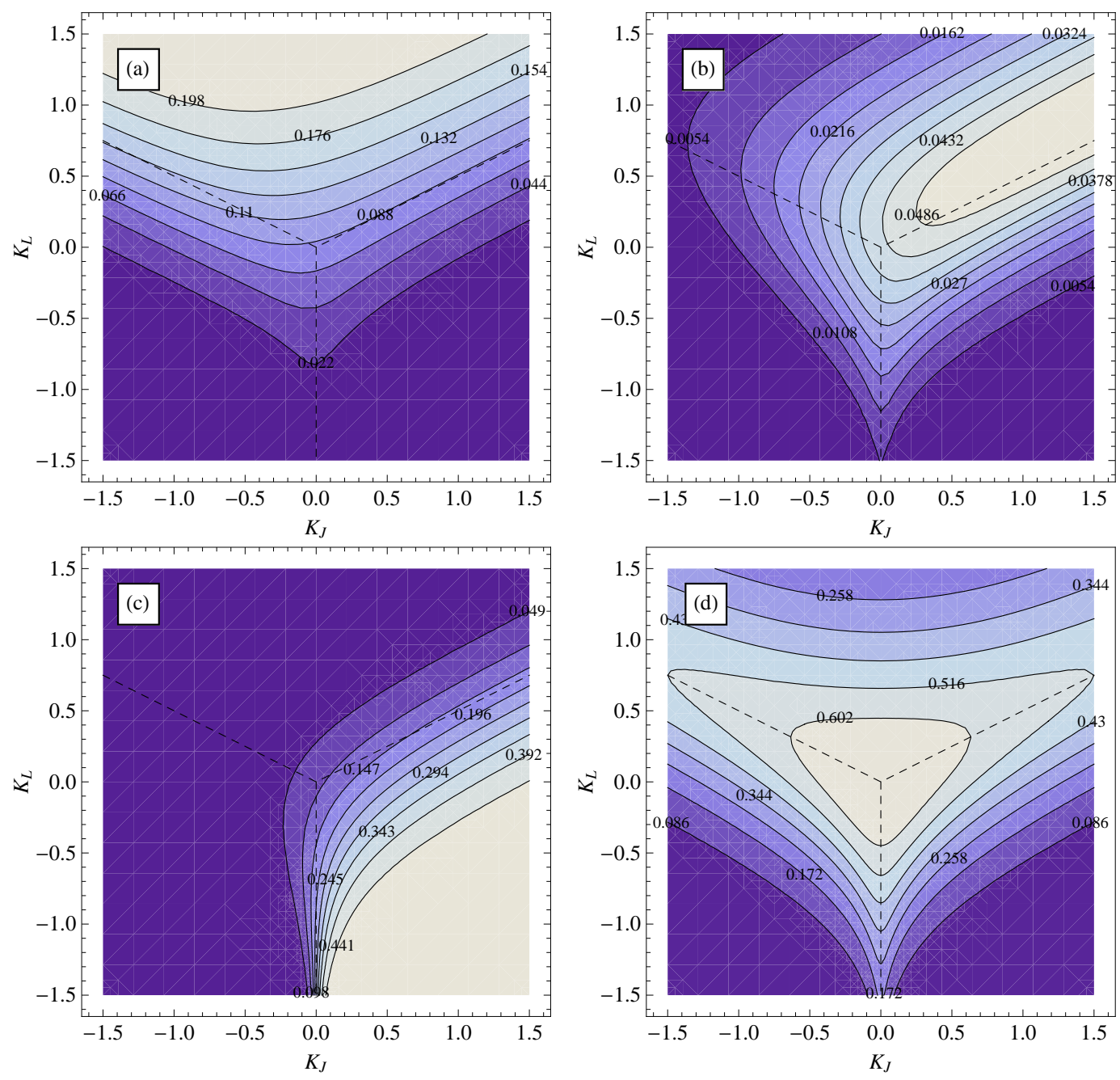

Figure 8. (Color online) Average number (per site) (a) $\left\langle\bar{N}_{1}\right\rangle$, (b) $\left\langle\bar{N}_{2}\right\rangle=\left\langle\bar{N}_{5}\right\rangle$, and (c) $\left\langle\bar{N}_{4}\right\rangle$ versus scaled variables $K_{J}, K_{L}$. The corresponding plot for $\left\langle\bar{N}_{3}\right\rangle$ is as shown in figure 7 (a). The graphs of $\left\langle\bar{N}_{3}\right\rangle$ and $\left\langle\bar{N}_{4}\right\rangle$ are not mirror images of each other. The graph of $\left\langle\bar{N}_{1}\right\rangle+\left\langle\bar{N}_{2}\right\rangle$ is identical to the graph shown in figure 7 (b). Panel (d) shows the entropy $\bar{S} / k_{\mathrm{B}}$ per lattice site as function of the scaled variables $K_{J}, K_{L}$.

For given $J$ and $L$ the entropy is a smooth and monotonously increasing function of $T$. Magnetic shortrange order (one of three kinds) establishes itself gradually and turns into long-range order at $T=0$. The thermal fluctuations are stronger at $L>0$ than at $L<0$ if $J \neq 0$ owing to competing nn and nnn couplings. $\bar{S} / k_{\mathrm{B}}$ decreasing smoothly from $\ln 2 \simeq 0.693$ at $T=\infty$ to zero at $T=0$ for all parameter combinations except $|J|=2 L$. Here $\bar{S} / k_{\mathrm{B}}$ approaches $\ln ([1+\sqrt{5}] / 2) \simeq 0.481$ in the low- $T$ limit. The implied ground-state degeneracy has a natural yet different interpretation in the context of each set of statistically interacting particles as shown.

\footnotetext{
${ }^{7}$ Alternatively derived from (13) via $S=k_{\mathrm{B}} \mathrm{d} T \ln Z_{N} / \mathrm{d} T$.
} 


\section{Structures from interactions}

In this work we have investigated structures caused by interactions in different ways. On a small scale, particles of various shapes are assembled from building blocks with minimal structure (Ising spins) by the $n n$ and nnn couplings of Hamiltonian (1). These particles with structures over the range of a few lattice sites are free of any inter-particle binding forces, not merely at low density but also at high density. Nevertheless, by virtue of their specific shapes, these particles are apt to assemble further structures on intermediate and large scales in the form of positional ordering of various kinds.

On an intermediate scale we have described the assembly of clusters of compacts, a process driven entirely by shapes and limited space. We have also described host particles consisting of two amphiphilic parts bracketing uniform arrays of tags or disordered mixtures of tags and hybrids, thus assuming the role of a surfactant or a membrane. Host particles are akin to micelles in this context. On a larger scale we have described the formation of macroscopically ordered patterns of particles from one or two species in a crowded environment. The type of ordering is determined solely by the energies, shapes, and sizes of the particles.

The structures stabilized on the smallest scale by the coupled Ising spins depend on our choice of reference state (pseudo-vacuum), hence the three sets of particle species in tables 1, 3. 5. However, in any given equilibrium state, the same large-scale ordering tendencies are produced by particles from different sets, i.e. by particles with different shapes, sizes, and energies.

The methodology developed here and in reference [8] has natural applications in research areas of strong current interest including jamming of granular matter in narrow channels [17] and DNA overstretching [18, 19].

\section{References}

1. Ashcroft N.W., Mermin N.D., Solid State Physics. Harcourt, New York, 1976.

2. Korepin V.E., Bogoliubov N.M., Izergin A.G., Quantum Inverse Scattering Method and Correlation Functions. Cambridge University Press, 1993.

3. Takahashi M., Thermodynamics of One-Dimensional Solvable Models. Cambridge University Press, 1999.

4. Sutherland B., Beautiful Models: 70 Years of Exactly Solved Quantum Many-Body Problems. World Scientific, Singapore, 2004.

5. Essler F.H.L., Frahm H., Göhmann F., Klümper A., Korepin V.E., The One-Dimensional Hubbard Model. Cambridge University Press, 2005.

6. Lu P., Vanasse J., Piecuch C., Karbach M., Müller G., J. Phys. A: Math. Theor., 2008, 41, 265003; doi $10.1088 / 1751-8113 / 41 / 26 / 265003$

7. Lu P., Müller G., Karbach M., Condens. Matter Phys., 2009, 12, 381; doi 10.5488/CMP.12.3.381

8. Liu D., Lu P., Müller G., Karbach M., Phys. Rev. E, 2011, 84, 021136; doi 10.1103/PhysRevE.84.021136

9. Igarashi J., Tonegawa T., J. Phys. Soc. Jpn., 1989, 58, 2147; doi 10.1143/JPSJ.58.2147. Igarashi J., Tonegawa T., Phys. Rev. B, 1989, 40, 756; doi 10.1103/PhysRevB.40.756

10. Ha Z.N.C., Haldane F.D.M., Phys. Rev. B, 1993, 47, 12459; doi 10.1103/PhysRevB.47.12459

11. Haldane F.D.M., Phys. Rev. Lett., 1991, 67, 937; doi 10.1103/PhysRevLett.67.937

12. Villain J., Physica B, 1975, 79, 1; doi 10.1016/0378-4363(75)90101-1. Ishimura N., Shiba H., Prog. Theor. Phys., 1980, 63, 743; doi 10.1143/PTP.63.743

13. Haldane F.D.M., Phys. Rev. Lett., 1991, 66, 1529; doi 10.1103/PhysRevLett.66.1529

14. Karbach M., Müller G., Wiele K., J. Phys. A: Math. Theor., 2008, 41, 205002; doi $10.1088 / 1751-8113 / 41 / 20 / 205002$

15. Wu Y.-S., Phys. Rev. Lett., 1994, 73, 922; doi 10.1103/PhysRevLett.73.922

16. Kramers H.A., Wannier G.H., Phys. Rev., 1941, 60, 252; doi 10.1103/PhysRev.60.252 Yeomans J.M., Statistical Mechanics of Phase Transitions. Clarendon Press, Oxford, 1992.

17. Bowles R.K., Saika-Voivod I., Phys. Rev. E, 2006, 73, 011503; doi 10.1103/PhysRevE.73.011503 Ashwin S.S., Bowles R.K., Phys. Rev. Lett., 2009, 102, 235701; doi 10.1103/PhysRevLett.102.235701

18. Vladescu I.D., McCauley M.J., Rouzina I., Williams M.C., Phys. Rev. Lett., 2005, 95, 158102; doi 10.1103/PhysRevLett.95.158102 van Mameren J., Proc. Natl. Acad. Sci. U.S.A., 2009, 106, 18231; doi 10.1073/pnas.0904322106

19. Sarkar A., Léger J.-F., Chatenay D., Marko J.F., Phys. Rev. E, 2001, 63, 051903; doi 10.1103/PhysRevE.63.051903. Léger J.-F., Romano G., Sarkar A., Robert J., Bourdieu L., Chatenay D., Marko J.F., Phys. Rev. Lett., 1999, 83, 1066; doi 10.1103/PhysRevLett.83.1066. 
20. Dobson J.F., J. Math. Phys., 1969, 10, 40; doi 10.1063/1.1664757

Stephenson J., Can. J. Phys., 1970, 48, 1724; doi 10.1139/p70-217

\title{
Взаємозв'язані мотиви і ландшафти ентропії статистично взаємодіючих частинок
}

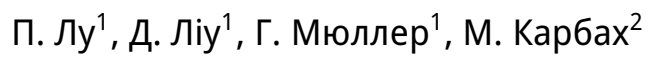 \\ 1 Фізичний факультет, Університет Род-Айленду, Кінгстон Род-Айленд 02881, США \\ 2 Фізичний факультет, Гірничий університет Вупперталя, 42097 Вупперталь, Німеччина
}

Використовуючи $s=1 / 2$ ізингівський ланцюжок з однорідною взаємодією найближчих і наступних за найближчими сусідів, побудовано систему незафіксованих частинок, які характеризуються мотивами шести послідовних локальних спінів. Взаємодія спінів спричиняє групування частинок, які, в свою чергу, не залежать від енергій взаємодії навіть при високій густині. Всі мікростани утворені конфігураціями частинок з одного із трьох різних наборів, які відповідають збудженням псевдовакуумів, що пов'язані із основними станами періодичності один, два і чотири. Мотиви частинок і елементи псевдовакууму об'єднуються в двох спільних вузлових змінних. Статистична взаємодія між частинками $є$ закодованою в узагальненому принципі Паулі, що описує як розміщення одної частинки змінює можливості для розміщення подальших частинок. В статистично механічному аналізі довільні енергії можуть ставитись у відповідність всім сортам частинок. Ентропія $\epsilon$ функцією заселеності частинок. Особливості статистичної взаємодії прозоро вбудовані в цей вираз. Енергії і структури частинок виключно визначають впорядкування при низьких температурах. За особливих умов частинки можуть бути замінені фундаментальнішими частинками з коротшими мотивами, що взаємозв'язуються однією спільною вузловою змінною. Зумовлені взаємодією структури виникають на двох рівнях: частинки з формами, які утворені взаємодіючими спінами, і тенденції до далекосяжного впорядкування частинок з формами, які статистично взаємодіють.

Ключові слова: принцип Паулі, частинки з формами, дробова статистика, модель Ізинга, солітони 


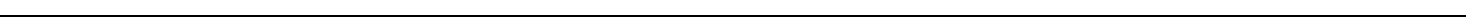

